\title{
Parameters of Viscous Heat-Conducting Gas-Air Flow in Mining Behind the Shock Front
}

\author{
Sergei Cherdantsev ${ }^{1,2, *}$, Yuri Filatov ${ }^{1}$, Pavel Shlapakov ${ }^{1}$, and Kirill Lebedev ${ }^{1}$ \\ ${ }^{1}$ Join-stock company "Scientific centre VOSTNII on industrial and ecological safety in mountain \\ industry" (JC "NC VOSTNII"), str. Institutskaya, 3, Kemerovo, 650002, Russia \\ ${ }^{2}$ T.F. Gorbachev Kuzbass State Technical University, 28 street Vesennyaya, Kemerovo, 650000, \\ Russian Federation
}

\begin{abstract}
With the transition of mining operations to deeper horizons, the safety of miners in coal mines is significantly reduced. This is mainly due to the manifestation of gas-dynamic and the thermische factors, the first of which include sudden emissions of coal and gas, gas venting, shock and detonation waves in the atmosphere of mine workings. The reasons for the thermal factors are the presence of dust-gas-air mixtures prone to chemical oxidation reactions of carbonaceous substances, and the formation of selfheating foci, which lead to changes in the temperature field in the atmosphere of mountain rocks and their surroundings, which significantly increases the probability of occurrence of deflagration and detonation processes. In this paper, the parameters of the shock front in a viscous heat-conducting gas-air flow formed by a sudden release of gas from an underground tank into a mine with a supersonic speed are determined. It is assumed that the movement of the gas-air mixture during the transition of the shock front is stationary, which made it possible to use the fundamental laws of conservation of mass, pulses and energy. The formulas determining the parameters of the gas-air flow are obtained, their graphs are constructed and some regularities of the flow parameters change behind the shock front depending on the initial Mach number and the Poisson adiabatic index are revealed.
\end{abstract}

\section{Introduction}

In the development of coal deposits on deep horizons, as a rule, there are gas-dynamic and thermophysical processes that adversely affect the safety and productivity of mining. Gasdynamic processes are caused by methane emissions into mine workings, which can occur at supersonic speed $[1,2]$.

The reason for negative thermo physical processes is dust-gas-air mixtures (PARS) capable of oxidation reactions accompanied by heat and mass transfer and the formation of self-heating centers [3,4], which lead to a change in the temperature field of rocks surrounding the treatment workings [5]. In the presence of ignition sources there is a

\footnotetext{
*Corresponding author: e-mail:svch01@yandex.ru
} 
deflagration of dust and gas mixtures in the atmosphere of mine workings [6, 7], which, under certain conditions, can go into detonation $[8,9]$.

In particular, the authors [9] argue that the cause of accidents in the development of flat coal seams is the ignition of methane-air mixture initiated by the centers of self-heating of coal and subsequent explosions in the developed space. The burning gas is carried out by an explosive wave in the treatment face and in the adjacent mine workings, which can cause detonation processes in coal dust, which are catastrophic.

It follows from the above that the most dangerous phenomena in coal mining enterprises are shock wave processes that initiate detonation waves, which are particularly serious accidents.

The classical theory of shock-wave processes in gases is constructed within the framework of the ideal gas model on the basis of three fundamental conservation laws: mass, pulses and energy. The main purpose of the theory is to find a solution to the problem of establishing the relationship between gas-dynamic and thermophysical parameters: speed, density, pressure and temperature of the gas [10]. For many special cases, the ideal gas model solutions to this problem are found.

However, under certain conditions, shock wave processes in mine workings are nonclassical problems. First of all, non-classical problems are the flow of gases in the mine workings with rough walls, creating a significant resistance to the movement of gas flows through the mine workings, which, in our opinion, leads to the need to take into account the viscous properties of the gas-air mixture.

To this we add that to date, the theory of shock-wave and detonation processes in rough pipes has not yet been developed in full [10]. Therefore, it seems to us that the problems of studying the structure, evolution and stability of shock waves in viscous and heatconducting gas-air mixtures in the atmosphere of mine workings are quite relevant. One of these tasks is discussed in this article.

\section{Statement of the problem about the parameters of a viscous heat-conducting gas flow in the mine workings behind the shock front}

Let the gas-air mixture flow out of the underground tank, which has a diffuser shape at the outlet to the mine workings, at a supersonic speed, causing the appearance of a shock wave, which, moving along the mine workings, forms a shock wave. It is required to determine the parameters of the mixture behind the shock wave front, assuming a gas-air mixture of viscous heat-conducting gas.

To solve the formulated problem, we use the fundamental conservation laws [10] described by the continuity equation

$$
\frac{\partial \rho}{\partial t}+\operatorname{div}(\rho \vec{v})=0 .
$$

equation of pulses

$$
\frac{\partial(\rho \vec{v})}{\partial t}=-\operatorname{div}(\rho \vec{v} \vec{v}+\Psi+\Phi) .
$$

and the energy transfer equation

$$
\frac{\partial}{\partial t}\left(\frac{\rho v^{2}}{2}+\rho \varepsilon\right)=-\operatorname{div}\left[\rho \vec{v}\left(\frac{v^{2}}{2}+i\right)-\Phi \vec{v}-\lambda \operatorname{grad} T\right] .
$$


Equations (1) - (3) form a system in which the desired values are: the velocity vector of the gas-air mixture $\vec{v}$, its pressure $p$, density $\rho$ and Kelvin temperature $T$. Other values in the system (1) - (3) are indicated by: $t-$ time, $\varepsilon$ and $i-$ respectively, the internal energy and enthalpy of the gas-air mixture, depending on the temperature $T ; \lambda-$ coefficient of thermal conductivity of the mixture; $\Psi=-p E-$ ball tensor containing hydrostatic pressure $\mathrm{p}$ and a single matrix $E ; \Phi-$ viscous stress tensor. The value included in equation (2) $\rho \vec{v} \vec{v}-$ is a dyad of vectors $\rho \vec{v}$ and $\vec{v}$ is a second rank tensor containing components $\left(\rho v_{i} v_{k}\right)$. Components of the tensor $\Phi$, we define by the formula [11]:

$$
\Phi_{i j}=\eta\left(\frac{\partial v_{i}}{\partial x_{k}}+\frac{\partial v_{k}}{\partial x_{i}}-\frac{2}{3} \delta_{i k} \frac{\partial v_{i}}{\partial x_{i}}\right)+\zeta \delta_{i k} \frac{\partial v_{i}}{\partial x_{i}}, \quad i, k=1,2,3,
$$

which takes into account the rule of summation by "dumb" indices, and the constants $\eta, \zeta$ - are the viscosity coefficients, the second of them is often called the "second" viscosity.

Shock-wave processes occur, as a rule, in a stationary mode [10]. Therefore, the derivatives of time $t$ in the system (1) - (3) are zero, whereby the divergence in all equations (1) - (3) is also zero and therefore the expressions in brackets under the sign of divergence do not depend on the coordinates $x, y, z$, as a result, we obtain the first integrals of equations (1) - (3)

$$
\rho \vec{v}=C_{1}, \quad \rho \vec{v} \vec{v}+\Psi+\Phi=C_{2}, \quad \rho \vec{v}\left(\frac{v^{2}}{2}+i\right)-\Phi \vec{v}-\lambda \operatorname{grad} T=C_{3} .
$$

The $\mathrm{x}$-axis is compatible with the output axis, along which the velocity component $v_{x}$, and the velocity components in the cross-sectional plane vu and $v_{z}$, obviously, $v_{y}=v_{z}=0$. In view of the above, the gas flow through the production will be one-dimensional, in which only one component of the nine components of the dyad $\rho \vec{v} \vec{v}$, the ball tensor $\Psi$ and the viscous stress tensor $\Phi$ take place, respectively

$$
p, \rho v_{x}^{2}, \Phi_{x x}=\left(\zeta+\frac{4}{3} \eta\right) \frac{d v_{x}}{d x}
$$

and so equality (5) is reduced to the form:

$$
\begin{gathered}
\rho v_{x}=C_{1}, \\
\rho v_{x}^{2}+p-\left(\zeta+\frac{4}{3} \eta\right) \frac{d v_{x}}{d x}=C_{2}, \\
\rho v_{x}\left(\frac{v_{x}^{2}}{2}+\frac{k}{k-1} \frac{p}{\rho}\right)-\left(\zeta+\frac{4}{3} \eta\right) v_{x} \frac{d v_{x}}{d x}-\lambda \frac{d T}{d x}=C_{3},
\end{gathered}
$$

where we took into account that the enthalpy is determined by the formula [10]: $i=k p /[(k-$ 1) $\rho$ ], in which $\mathrm{k}$ is an indicator of Poisson's adiabatic in an air-gas mixture.

The values of the constants $C_{1}, C_{2}, C_{3}$ in the right parts of the equations (6) - (8) are determined from the boundary conditions

$$
\left.\frac{d v_{x}}{d x}\right|_{x \rightarrow-\infty}=0,\left.\frac{d T}{d x}\right|_{x \rightarrow-\infty}=0,\left.\rho\right|_{x \rightarrow-\infty}=\rho_{0},\left.\quad v_{x}\right|_{x \rightarrow-\infty}=v_{x 0},\left.\quad T\right|_{x \rightarrow-\infty}=T_{0},
$$


meaning that at a sufficiently large distance from the zone of formation of the shock front all the derivatives of $x$ go to zero, and the magnitude $v_{x}, \rho, p, T$ are the initial value $v_{\mathrm{x} 0}, \rho_{0}$, $p_{0}, T_{0}$ in the undisturbed gas flow (at $\left.x \rightarrow-\infty\right)$.

Substituting (9) into the system (6) - (8), we find constant integrations

$$
C_{1}=\rho_{0} v_{x 0}, \quad C_{2}=\rho_{0} v_{x 0}^{2}+p_{0}, \quad C_{3}=\rho_{0} v_{x 0}\left(\frac{v_{x 0}^{2}}{2}+\frac{k}{k-1} \frac{p_{0}}{\rho_{0}}\right),
$$

taking into account which equations (6) - (8) take the form

$$
\begin{gathered}
\rho v_{x}=\rho_{0} v_{x 0}, \\
\rho v_{x}^{2}+p-\left(\zeta+\frac{4}{3} \eta\right) \frac{d v_{x}}{d x}=\rho_{0} v_{x 0}^{2}+p_{0}, \\
\rho v_{x}\left(\frac{v_{x}^{2}}{2}+\frac{k}{k-1} \frac{p}{\rho}\right)-\lambda \frac{d T}{d x}-\left(\zeta+\frac{4}{3} \eta\right) v_{x} \frac{d v_{x}}{d x}=\rho_{0} v_{x 0}\left(\frac{v_{x 0}^{2}}{2}+\frac{k}{k-1} \frac{p_{0}}{\rho_{0}}\right) .
\end{gathered}
$$

Dividing the first of the equations by $\rho_{0} v_{x 0}$, the second by $\rho_{0} v_{x 0}^{2}$, and the third by $\rho_{0} v_{x 0}^{3}$ and after simple but rather cumbersome transformations, we obtain the following system of equations

$$
\begin{gathered}
\bar{\rho} u=1, \\
u-1+\frac{1}{k \mathrm{M}_{0}^{2}}\left(\frac{\mathrm{T}}{u}-1\right)-K_{v} \frac{d u}{d x}=0, \\
u^{2}-1+\frac{2(\mathrm{~T}-1)}{(k-1) \mathrm{M}_{0}^{2}}-2 K_{v} u \frac{d u}{d x}-2 K_{T} \frac{d \mathrm{~T}}{d x}=0,
\end{gathered}
$$

in which: $u=v_{x} / v_{x 0}, \bar{\rho}=\rho / \rho_{0}, \mathrm{~T}=T / T_{0}$ - are respectively dimensionless velocity, density and temperature of the mixture.

Included in the system (10) - (12) values $K_{v}$ and $K_{T}$ are the parameters characterizing the viscosity and thermal conductivity of the gas-air mixture, and the value $\mathrm{M}_{0}$ is the Mach number:

$$
K_{v}=\frac{\zeta+4 / 3 \eta}{\rho_{0} v_{x 0}}, K_{T}=\frac{\lambda T_{0}}{\rho_{0} v_{x 0}^{3}}, \mathrm{M}_{0}=\frac{v_{x 0}}{a_{0}},
$$

where $a_{0}$ is the speed of sound in an undisturbed gas-air flow determined by the formula [10]: $a_{0}=\sqrt{k p_{0} / \rho_{0}}$.

$$
\begin{aligned}
& \text { At } x \rightarrow+\infty \\
& \qquad\left.\frac{d v_{x}}{d x}\right|_{x \rightarrow+\infty}=0,\left.\frac{d T}{d x}\right|_{x \rightarrow+\infty}=0,\left.\rho\right|_{x \rightarrow+\infty}=\rho_{2},\left.u\right|_{x \rightarrow+\infty}=u_{2},\left.\quad T\right|_{x \rightarrow+\infty}=T_{2}
\end{aligned}
$$

equations (10) - (12) are converted to form 


$$
\bar{\rho}_{2} u_{2}=1, u_{2}-1+\frac{1}{k \mathrm{M}_{0}^{2}}\left(\frac{\mathrm{T}_{2}}{u_{2}}-1\right)=0, u_{2}^{2}-1+\frac{2\left(\mathrm{~T}_{2}-1\right)}{(k-1) \mathrm{M}_{0}^{2}}=0 .
$$

From the third equation of the system (14) we Express the temperature $T_{2}$ through the velocity $u_{2}$

$$
\mathrm{T}_{2}=\frac{2-\left(u_{2}^{2}-1\right)(k-1) \mathrm{M}_{0}^{2}}{2}
$$

and substituting the second equation (14), we obtain a square equation with respect to the velocity $u_{2}$

$$
u_{2}^{2}-\frac{2\left(k \mathrm{M}_{0}^{2}+1\right)}{\mathrm{M}_{0}^{2}(k+1)} u_{2}+\frac{(k-1) \mathrm{M}_{0}^{2}+2}{\mathrm{M}_{0}^{2}(k+1)}=0
$$

whose roots are

$$
u_{2(1)}=1, u_{2}=\frac{\mathrm{M}_{0}^{2}(k-1)+2}{\mathrm{M}_{0}^{2}(k+1)} .
$$

The first root $u_{2(1)}$ is trivial because it is equal to the velocity of the mixture $\left.u_{0}\right|_{x \rightarrow-\infty}=1$, which is why the shock wave is not formed. The second root $u_{2}$ determines the velocity of the gas-air mixture behind the shock front.

Knowing the velocity $u_{2}$, from the first equality (14) we determine the relative density of the mixture

$$
\bar{\rho}_{2}=\frac{1}{u_{2}}=\frac{\mathrm{M}_{0}^{2}(k+1)}{\mathrm{M}_{0}^{2}(k-1)+2},
$$

using the formula (15) we find the temperature behind the shock front

$$
\mathrm{T}_{2}=\frac{\left[2 k \mathrm{M}_{0}^{2}-(k-1)\right]\left[2+(k-1) \mathrm{M}_{0}^{2}\right]}{\mathrm{M}_{0}^{2}(k+1)^{2}},
$$

and using the Mendeleev - Clapeyron law $p_{2}=\rho_{2} R T_{2}$ and formulas (17), (18) we determine the dimensionless pressure $\bar{p}_{2}$ behind the shock front:

$$
\bar{p}_{2}=\frac{2 k \mathrm{M}_{0}^{2}-(k-1)}{k+1} .
$$

On fig. $1-2$ shows graphs of the dependence of the required parameters on the Mach number $\mathrm{M}_{0}$, constructed by formulas (16) - (19), for a number of values of the Poisson's adiabatic index $k$. 

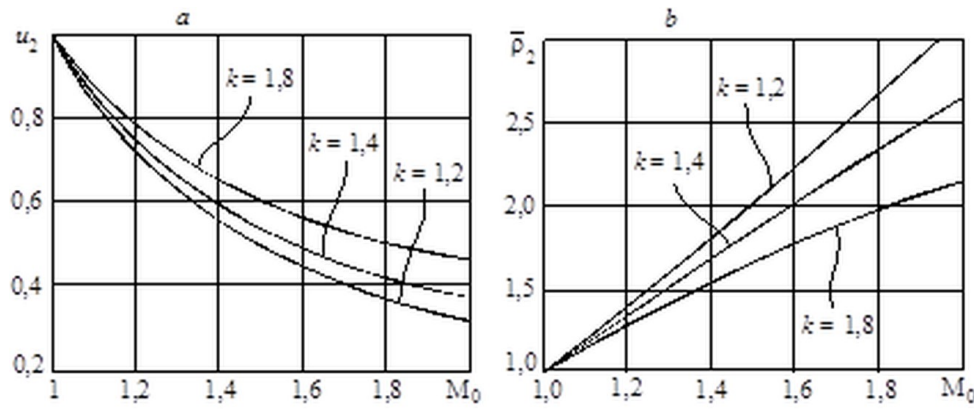

Fig. 1. Graphs of the dependence of the velocity $u_{2}(a)$ and density $\bar{\rho}_{2}(b)$ of the mixture behind the shock front on the Mach number for a number of values of the Poisson adiabatic index.

Analyzing the figures, we note that the speed of the gas-air mixture flowing from the underground tank drops sharply, especially at small values of $k$. In this case, the function $u_{2}\left(\mathrm{M}_{0}\right)$ decreases monotonically, and its graphs for a number of $k$ values represent concave curves (Fig. 1a). On the contrary, the function increases monotonically, and the more significant the smaller the Poisson adiabatic index (Fig. 1b).

Other parameters of the air-gas flow: pressure and temperature will also melt (Fig. 2). Moreover, this is more typical for a function $\bar{p}_{2}\left(\mathrm{M}_{0}\right)$ whose graphs at different values of $k$ are concave curves monotonically increasing with increasing Mach number $\mathrm{M}_{0}$.

Compared to pressure, the temperature increases less significantly, and the graphs of the $\mathrm{T}_{2}\left(\mathrm{M}_{0}\right)$ function differ little from straight lines.
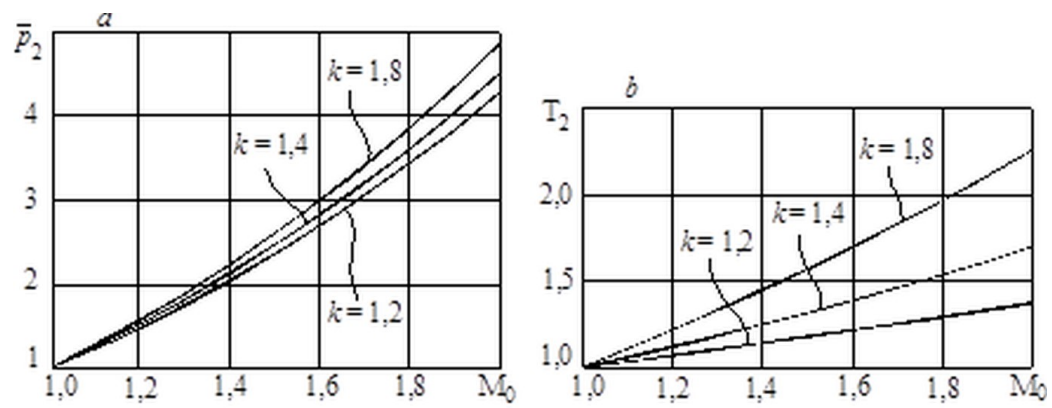

Fig. 2. Graphs of pressure $\bar{p}_{2}(a)$ and temperature $\mathrm{T}_{2}(b)$ of the mixture behind the shock front of the Mach number for a number of values of the Poisson's adiabatic index.

To the above, we add that large values of pressure and temperature at a fixed Mach number occur at large values of the Poisson's adiabate index, whose influence on the pressure in the mixture is less significant than on the temperature.

\section{Conclusion}

1. The parameters of the gas-air mixture behind the shock front are determined.

2. Graphs of the dependence of velocity, density, pressure and temperature on the Mach number $\mathrm{M}_{0}$ for a number of values of the Poisson's adiabatic index $k$ are constructed.

3 Analysis of the graphs showed that in the collision of the gas-air flow coming out of the underground tank with the sedentary air of the mine workings:

a) the speed of the gas-air mixture drops sharply, especially at small values of $k$, and the density, pressure and temperature increase; 
b) the lower the Poisson's adiabatic index, the higher the density of the gas-air flow, but the lower the pressure and temperature at a fixed Mach number.

\section{References}

1. M. A. Liberman, A. L. Velikovich, J. Plasma Phys, 26, 59 (1981)

2. J. Howard, B. W. James, A. R. Law, Ibid, 27, 277 (1984)

3. A. I. Chanyshev, Journal of Mining Science, 48:4, 660 (2012)

4. V. N. Oparin, T. A. Kiryaeva, V. Yu. Gavrilov, Yu. Yu. Tanashev, V. A. Bolotov, Journal of Mining Science, 52:3, 576 (2016)

5. S. V. Cherdantsev, P. A. Shlapakov, A. Yu. Erastov, S. A. Khaymin, K. S. Lebedev, V. V. Kolykhalov, E. A. Shlapakov, IOP Conference Series: Earth and Environmental Science, 206, 012006, (2018)

6. S. R. Rockwell, R. Scott, Ali S. Rangwala, Combustion and Flame, 160, 635 (2013)

7. S. V. Cherdantsev, Hi Un Li, Yu. M. Filatov, D. V. Botvenko, P. A. Shlapakov, V. V. Kolykhalov, Journal of Mining Science, 54:2, 339 (2018)

8. J. M. Austin, J. E. Shepherd, Combust. Flame, 132:1-2, 73 (2003)

9. M. V. Kurlenya, V. A. Skritsky, Journal of Mining Science, 53:5, 861 (2017)

10. A. Bartlma, Gasdynamik der verbrennung. Deutsche forschungs and versuchsanstalt (Institut fur Reaktionskinetik, Stuttgart, 1975)

11. Mase G. E. Theory and problems of continuum mechanics. (Lomann Press., London. 1970) 